\title{
NEW COMPARISONS FOR LOCAL QUANTITIES OF THE TWO-DIMENSIONAL HUBBARD MODEL
}

\author{
ADOLFO AVELLA AND FERDINANDO MANCINI \\ Dipartimento di Fisica "E.R. Caianiello" - Unità INFM di Salerno \\ Università degli Studi di Salerno, I-84081 Baronissi (SA),Italy \\ E-mail: avella@sa.infn.it
}

\begin{abstract}
We have compared the results of our approximation scheme, the composite operator method, for the double occupancy and the internal energy of the two-dimensional Hubbard model with numerical data obtained by means of the Lanczos ${ }^{1}$ and quantum Monte Carlo schemes ${ }^{2}$. The agreement is very good at both half-filling and away from it showing how reliable is the approximation scheme.
\end{abstract}

\section{Introduction}

The relevance of the Hubbard model ${ }^{3}$ as minimal model for describing the high$\mathrm{T}_{c}$ cuprate superconductors ${ }^{4}$ and, generally, many strongly correlated materials ${ }^{5}$ is very well established. The model is thought to have a very rich phenomenology: a variety of spin and charge orderings ${ }^{6}$ (ferro- and antiferro- magnetic included), Mott transitions (of the Slater, Heisenberg and Hubbard types) driven by both filling and coupling 7,8 , non-Fermi liquid dynamics, superconductivity caused by: magnons, static and dynamic charge ordering, proximity to quantum critical points of different origin. In particular, strong antiferromagnetic correlations are present at half-filling (and for low doping) and low temperatures ${ }^{6}$.

According to this, it is very relevant to have reliable solutions of this model for the variety of boundary conditions under which it can be studied. Actually, we know the exact solution only in one dimension thanks to the Bethe Ansatz ${ }^{9}$. Some other few exact results are known in quite special limits, but the more relevant questions are still unsolved as the exact solution in two dimensions (which seems to be the case for the majority of the emergent highly interacting materials) is still missing. Owing to this, a huge number of approximation schemes have been proposed since the very beginning (Hubbard presented the model together with an approximate solution known today as Hubbard I) and their reliability is still under test as many physical interpretations are based on the results obtained by them.

In the last decades we have seen the birth and development of many numerical methods (exact diagonalization, Lanczos, quantum Monte Carlo, ...) for studying finite clusters of bigger and bigger size. The results of these numerical methods are extremely important for the development of analytical schemes as they provide the possibility to execute unbiased tests. The numerical data can be interpreted as the experimental results relative to a specific model and any comparison with an analytical approximation scheme can be directly contrasted with its reliability. On the contrary, comparisons with real experimental data suffer of an high degree of uncertainty regarding the capability of the chosen model to capture the essential physical of the material under analysis.

In this last decade, we have been developing an approximation scheme, namely the composite operator method ${ }^{8,10-13}$, which proved to be quite powerful to de- 
scribe the physics of many correlated models and the properties of some materials. The main peculiarities of this method are: the use of symmetry constraints (Pauli principle and Ward-Takahashi identities) to fix the representation where the Green's functions are realized, the freedom in choosing the composite fields for the operatorial basis around which we wish to construct perturbative solutions. The algebra imposes many relations between operators which have to be fulfilled also at the level of averages. According to this, it is possible to individuate a set of self-consistent equations which allows to fix the unknown parameters coming in the calculations and, consequently, to instruct the solution regarding the Hilbert space in which the composite operators live (i.e., the representation). In particular, we see the unknown parameters as a necessity and not as an accident as many other approximation schemes do. In the last years, we have acquired much experience in choosing the composite operators more effective in describing the physical property of a system and we have collected a certain number of recipes which can be used for specific class of models.

In this manuscript, we wish to present some new comparisons between numerical results and composite operator method ones for two local quantities: the double occupancy and the internal energy.

\section{Results}

The Hubbard model is described by the following Hamiltonian

$$
H=\sum_{\mathbf{i} \mathbf{j}}\left(t_{\mathbf{i j}}-\mu \delta_{\mathbf{i j}}\right) c^{\dagger}(i) c(j)+U \sum_{\mathbf{i}} n_{\uparrow}(i) n_{\downarrow}(i)
$$

where $c^{\dagger}(i)=\left(c_{\uparrow}^{\dagger}(i), c_{\downarrow}^{\dagger}(i)\right)$ is the electronic creation operator in spinorial notation at the site $\mathbf{i}[i=(\mathbf{i}, t)]$ and $n_{\sigma}(i)=c_{\sigma}^{\dagger}(i) c_{\sigma}(i)$ is the number operator for spin $\sigma$ at the site $\mathbf{i} ; \mu$ is the chemical potential and $U$ is the on-site Coulomb repulsion.

The matrix $t_{\mathbf{i j}}$ describes the nearest-neighbor hopping; in the $2 \mathrm{D}$ case we have $t_{\mathbf{i j}}=-4 t \alpha_{\mathbf{i j}}$, where

$$
\alpha_{\mathbf{i j}}=\frac{1}{N} \sum_{\mathbf{k}} e^{\mathrm{i} \mathbf{k}(\mathbf{i}-\mathbf{j})} \alpha(\mathbf{k})
$$

is the projector on the nearest-neighbor sites and $\alpha(\mathbf{k})=\frac{1}{2}\left[\cos \left(k_{x} a\right)+\cos \left(k_{y} a\right)\right]$ and $a$ is the lattice parameter.

We choose the following fermionic basis

$$
\Psi(i)=\left(\begin{array}{c}
\xi(i) \\
\eta(i)
\end{array}\right)
$$

where $\xi(i)=[1-n(i)] c(i)$ and $\eta(i)=n(i) c(i)$ are the Hubbard operators. $\Psi(i)$ satisfies the following equation of motion

$$
J(i)=\mathrm{i} \frac{\partial}{\partial t} \Psi(i)=\left(\begin{array}{c}
-\mu \xi(i)-4 t c^{\alpha}(i)-4 t \pi(i) \\
-(\mu-U) \eta(i)+4 t \pi(i)
\end{array}\right)
$$


where $c^{\alpha}(\mathbf{i}, t)=\sum_{\mathbf{j}} \alpha_{\mathbf{i j}} c(\mathbf{j}, t)$ and $\pi(i)=\frac{1}{2} \sigma^{\mu} n_{\mu}(i) c^{\alpha}(i)+c(i)\left[c^{\dagger \alpha}(i) c(i)\right]$. $n_{\mu}(i)=c^{\dagger}(i) \sigma_{\mu} c(i)$ are the charge $(\mu=0)$ and spin $(\mu=1,2,3)$ density operators, with $\sigma_{\mu}=(1, \vec{\sigma}), \sigma^{\mu}=(-1, \vec{\sigma})$ and $\vec{\sigma}$ are the Pauli matrices.

Let us project the source $J(i)$ on the chosen basis

$$
J(\mathbf{i}, t) \cong \sum_{\mathbf{j}} \varepsilon(\mathbf{i}, \mathbf{j}) \Psi(\mathbf{j}, t)
$$

Accordingly, the energy matrix $\varepsilon(\mathbf{i}, \mathbf{j})$ is defined through the equation

$$
m(\mathbf{i}, \mathbf{j})=\sum_{\mathbf{l}} \varepsilon(\mathbf{i}, \mathbf{l}) I(\mathbf{l}, \mathbf{j})
$$

where the $m$-matrix and the normalization matrix $I$ have the following definitions

$$
\begin{aligned}
& m(\mathbf{i}, \mathbf{j})=\left\langle\left\{J(\mathbf{i}, t), \Psi^{\dagger}(\mathbf{j}, t)\right\}\right\rangle \\
& I(\mathbf{i}, \mathbf{j})=\left\langle\left\{\Psi(\mathbf{i}, t), \Psi^{\dagger}(\mathbf{j}, t)\right\}\right\rangle
\end{aligned}
$$

After Eq. (5), the Fourier transform of the thermal single-particle retarded Green's function $G(i, j)=\left\langle R\left[\Psi(i) \Psi^{\dagger}(j)\right]\right\rangle$ satisfies the following equation

$$
[\omega-\varepsilon(\mathbf{k})] G(\mathbf{k}, \omega)=I(\mathbf{k})
$$

The straightforward application of this scheme $e^{8,10-13}$ gives that, in the paramagnetic phase, $I(\mathbf{k})$ has diagonal form with $I_{11}=1-n / 2$ and $I_{22}=n / 2$ $\left(\left\langle n_{\sigma}(i)\right\rangle=\frac{n}{2}\right)$ and that the $m$-matrix depends on three parameters: the chemical potential $\mu$ and two correlators

$$
\begin{aligned}
& \Delta=\left\langle\xi^{\alpha}(i) \xi^{\dagger}(i)\right\rangle-\left\langle\eta^{\alpha}(i) \eta^{\dagger}(i)\right\rangle \\
& p=\frac{1}{4}\left\langle n_{\mu}^{\alpha}(i) n_{\mu}(i)\right\rangle-\left\langle\left[c_{\uparrow}(i) c_{\downarrow}(i)\right]^{\alpha} c_{\downarrow}^{\dagger}(i) c_{\uparrow}^{\dagger}(i)\right\rangle
\end{aligned}
$$

The three parameters $\mu, \Delta$ and $p$ are functions of the external parameters $n, T$ and $U$ and can be determined self-consistently through a set of three coupled equations

$$
\left\{\begin{array}{l}
n=2\left[1-\left\langle c(i) c^{\dagger}(i)\right\rangle\right] \\
\Delta=\left\langle\xi^{\alpha}(i) \xi^{\dagger}(i)\right\rangle-\left\langle\eta^{\alpha}(i) \eta^{\dagger}(i)\right\rangle \\
\left\langle\xi(i) \eta^{\dagger}(i)\right\rangle=0
\end{array}\right.
$$

The first equation fixes the particle number, the second one comes from the definition of $\Delta$ and the third one assures that the solution respects the Pauli principle (i.e., the local algebra) ${ }^{14}$.

In Fig. 1 we report the behavior of the internal energy per site $E_{s}=\langle H\rangle / N$ as a function of the Coulomb interaction $U$ at half-filling and $n=8 / 9$. The Lanczos numerical data have been taken from Ref. ${ }^{1}$. The agreement at half-filling is very good. We have got an exceptionally good agreement at half-filling for the onedimensional Hubbard model too ${ }^{15}$. In that case, we have compared our results with the exact Bethe ansatz solution. Away from half-filling, the agreement is less satisfactory, but, as we will see from the double occupancy comparison, the difference is quite constant as function of $U$ and can be due to finite size effects, as surely is the difference at $U=0$.

Numerical: submitted to World Scientific on November 20, 2018 


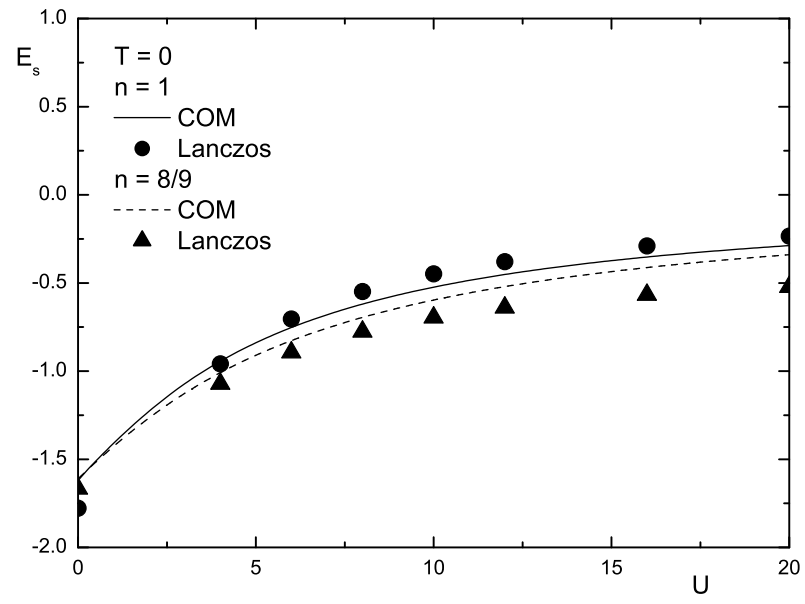

Figure 1. Internal energy per site $E_{s}$ as a function of the Coulomb interaction $U$ for $T=0$ and $n=1$ and $8 / 9$. The Lanczos data are taken from Ref. ${ }^{1}$.

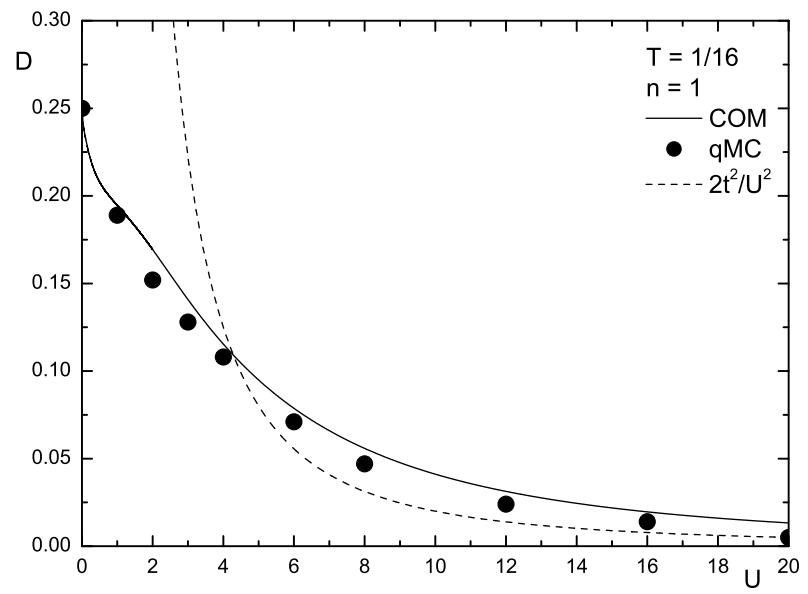

Figure 2. Double occupancy $D$ as a function of the Coulomb interaction $U$ for $T=1 / 16$ and $n=1$. The quantum Monte Carlo data are taken from Ref. ${ }^{2}$.

The double occupancy $D=\left\langle n_{\uparrow} n_{\downarrow}\right\rangle$, which can be computed by means of thermodynamics through the following formula $D=\frac{\partial F}{\partial U}$ ( $F$ is the free energy; for $T=0$ we have $D=\frac{\partial E_{s}}{\partial U}$ ), is reported as a function of the Coulomb interaction $U$ in Fig. 2 for $T=1 / 16$ and $n=1$ and in Fig. 3 for $T=0$ and $n=8 / 9$. The Lanczos numerical data have again been taken from Ref. ${ }^{1}$; the quantum Monte Carlo ones from Ref. ${ }^{2}$. The agreement is very good for both values of filling. For half-filling, we have also reported the limiting behavior of our solution: $D \stackrel{U \rightarrow \infty}{\longrightarrow} d \frac{t^{2}}{U^{2}}$ where $d$ is the spatial dimension of the system ${ }^{16}$. The numerical data seem to follow this behavior 


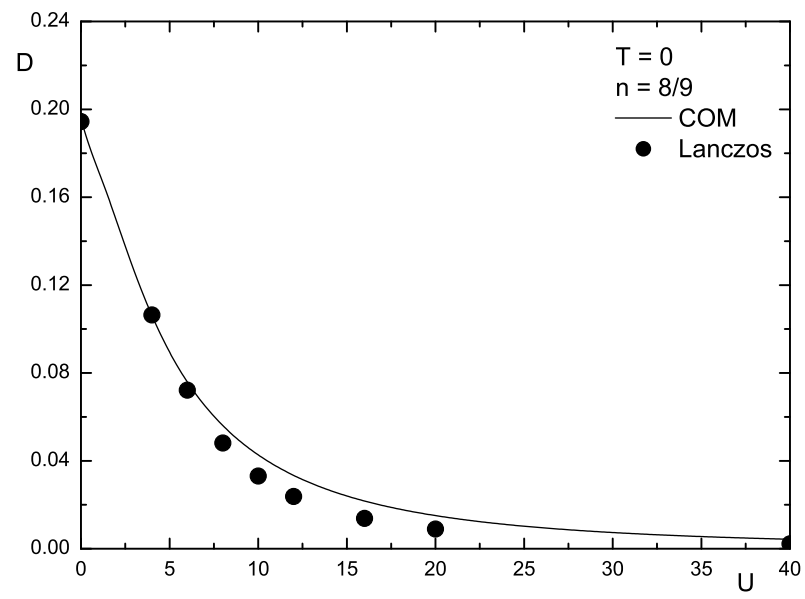

Figure 3. Double occupancy $D$ as a function of the Coulomb interaction $U$ for $T=0$ and $n=8 / 9$. The Lanczos data are taken from Ref. ${ }^{1}$.

also for not so large value of the Coulomb repulsion. This is probably due to finite size effects. For $n=8 / 9$, the very good agreement supports our interpretation of the comparison for the corresponding energy.

\section{Conclusions}

The positive comparisons with the Bethe ansatz exact solution for the onedimensional case and with the numerical results for the two-dimensional one show how reliable is our approximation scheme to study the Hubbard model. In particular, the agreement reported in this manuscript with the Lanczos and the quantum Monte Carlo data at both half-filling and away from it is very good and shows our capability to properly describe both the charge and the spin fluctuation scales of energy.

\section{References}

1. F. Becca, A. Parola, and S. Sorella. Phys. Rev. B, 61:16287, 2000.

2. S. R. White, D. J. Scalapino, R. L. Sugar, E. Y. Loh, J. E. Gubernatis, and R. T. Scalettar. Phys. Rev. B, 40:506, 1989.

3. J. Hubbard. Proc. Roy. Soc. A, 276:238, 1963; 277:237, 1964; 281:401, 1964; 285:542, 1965.

4. P.W. Anderson. Science, 235:1196, 1987.

5. P. Fulde. Electron Correlations in Molecules and Solids. Springer-Verlag, third edition, 1995.

6. E. Dagotto. Rev. Mod. Phys., 66:763, 1994.

7. F. Gebhard. The Mott Metal-Insulator Transition: Models and Methods. Springer, Berlin, 1997. 
8. A. Avella, F. Mancini, and R. Münzner. Phys. Rev. B, 63:245117, 2001.

9. E. H. Lieb and F. Y. Wu. Phys. Rev. Lett., 20:1445, 1968.

10. F. Mancini, S. Marra, and H. Matsumoto. Physica C, 244:49, 1995; 250:184, 1995; 252:361, 1995.

11. A. Avella, F. Mancini, D. Villani, L. Siurakshina, and V. Y. Yushankhai. Int. J. Mod. Phys. B, 12:81, 1998.

12. F. Mancini, D. Villani, and H. Matsumoto. Phys. Rev. B, 57:6145, 1998.

13. V. Fiorentino, F. Mancini, E. Zasinas, and A.F. Barabanov. Phys. Rev. B, 64:214515, 2001.

14. F. Mancini and A. Avella. The pauli principle and the green's function formalism. cond-mat/0006377.

15. A. Avella, F. Mancini, and M.M. Sánchez. Europhys. Lett., 44:328, 1998.

16. F. Mancini and V. Turkowski. Acta Phys. Pol. A, 101:505, 2002. 